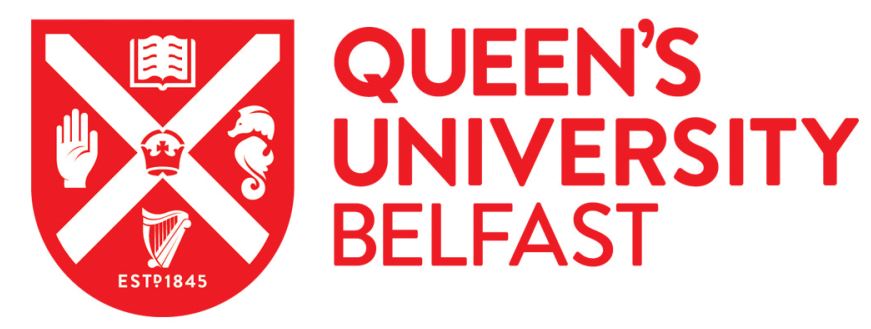

\title{
Time-resolved four-wave-mixing spectroscopy for inner-valence transitions
}

Ding, T., Ott, C., Kaldun, A., Blatterman, A., Meyer, K., Stooss, V., Rebholz, M., Birk, P., Hartmann, M., Brown, A., Van Der Hart, H., \& Pfeifer, T. (2016). Time-resolved four-wave-mixing spectroscopy for inner-valence transitions. Optics Letters, 41(4), 709-712. https://doi.org/10.1364/OL.41.000709

\section{Published in: \\ Optics Letters}

\section{Document Version:}

Peer reviewed version

Queen's University Belfast - Research Portal:

Link to publication record in Queen's University Belfast Research Portal

\section{Publisher rights}

(C) 2016 Optical Society of America

One print or electronic copy may be made for personal use only. Systematic reproduction and distribution, duplication of any material in this paper for a fee or for commercial purposes, or modifications of the content of this paper are prohibited.

\section{General rights}

Copyright for the publications made accessible via the Queen's University Belfast Research Portal is retained by the author(s) and / or other copyright owners and it is a condition of accessing these publications that users recognise and abide by the legal requirements associated with these rights.

Take down policy

The Research Portal is Queen's institutional repository that provides access to Queen's research output. Every effort has been made to ensure that content in the Research Portal does not infringe any person's rights, or applicable UK laws. If you discover content in the Research Portal that you believe breaches copyright or violates any law, please contact openaccess@qub.ac.uk. 


\title{
Time-resolved four-wave-mixing spectroscopy for inner-valence transitions
}

\author{
Thomas Ding, ${ }^{1,}$ * Christian Ott, ${ }^{1,2}$ Andreas Kaldun, ${ }^{1}$ Alexander \\ Blättermann, ${ }^{1}$ Kristina Meyer, ${ }^{1}$ Veit Stooss, ${ }^{1}$ Marc Rebholz, ${ }^{1}$ Paul Birk, ${ }^{1}$ \\ Maximilian hartmann, ${ }^{1}$ Andrew Brown, ${ }^{4}$ Hugo Van Der Hart, ${ }^{4}$ AND \\ THOMAS PfEIFER, ${ }^{1,3}$ \\ ${ }^{1}$ Max-Planck-Institut für Kernphysik, Saupfercheckweg 1, 69117 Heidelberg, Germany \\ ${ }^{2}$ Chemistry Department, University of California, Berkeley, California, 94720, USA \\ ${ }^{3}$ Centre for Quantum Dynamics, Ruprecht-Karls-Universität Heidelberg, 69120 Heidelberg, Germany \\ ${ }^{4}$ Centre for Theoretical Atomic, Molecular and Optical Physics, Queen's University Belfast, Belfast BT7 1NN, UK \\ *Corresponding author: thomas.ding@mpi-hd.mpg.de
}

Received XX Month XXXX; revised XX Month, XXXX; accepted XX Month XXXX; posted XX Month XXXX (Doc. ID XXXXX); published XX Month XXXX

Non-collinear four-wave mixing (FWM) techniques at near-infrared (NIR), visible, and ultraviolet frequencies have been widely used to map vibrational and electronic couplings, typically in complex molecules. However, correlations between spatially localized inner-valence transitions among different sites of a molecule in the extreme ultraviolet (XUV) spectral range have not been observed yet. As an experimental step towards this goal we perform time-resolved FWM spectroscopy with femtosecond NIR and attosecond XUV pulses. The first two pulses (XUV-NIR) coincide in time and act as coherent excitation fields, while the third pulse (NIR) acts as a probe. As a first application we show how coupling dynamics between odd- and even-parity inner-valence excited states of neon can be revealed using a two-dimensional spectral representation. Experimentally obtained results are found to be in good agreement with $a b$ initio time-dependent R-matrix calculations providing the full description of multi-electron interactions, as well as few-level model simulations. Future applications of this method also include site-specific probing of electronic processes in molecules.

OCIS codes: (190.0190) Nonlinear optics; (020.3690) Line shapes and shift; (300.2570) Four-wave mixing; (300.6240) Spectroscopy, coherent transient; (320.7110) Ultrafast nonlinear optics; (300.1030) Absorption.

With the development of coherent femtosecond-duration laser pulses nonlinear (third-order) four-wave-mixing (FWM) spectroscopy has become a versatile tool for the investigation of ultrafast dynamics in molecules and other material samples. A large body of experimental approaches for time-resolved molecular spectroscopy has been developed based on FWM phenomena, such as photon echo [1], coherent anti-Stokes Raman scattering [2], transient grating [3], and many more. The spectroscopically most comprehensive implementation of FWM is the so-called two-dimensional spectroscopy (2DS) with three temporally independent pulses which allows the correlation of excitation- and nonlinear-response spectra to directly measure couplings between quantum states [4,5]. 2DS in the infrared, visible, and ultraviolet spectral ranges has enabled the exploration of vibrational [6], electronic [7] and vibronic [8] coupling dynamics in complex molecular systems. The extension of 2DS into the extreme ultraviolet (XUV) and X-ray spectral regions aims to map coupling dynamics between spatially localized inner-valence or core transitions among different sites of a quantum system. It has been theoretically discussed by Mukamel and coworkers [9,10] and is a long-awaited goal. In principle, modern free-electron laser (FEL) and high-harmonic generation (HHG) based coherent XUV and X-ray light sources can provide appropriate pulses for 2DS experiments, but key challenges are both the increased technical demands to create appropriate multipulse geometries in this photon energy range, as well as the intrinsically low photon flux of laboratory based sources. Nevertheless, first steps are being made, as demonstrated in recent experiments that explore NIR- and XUV-induced transient gratings that emphasize both the spectroscopic element selectivity [11] and the enhanced spatial resolution [12] capabilities of the XUV domain.

Meanwhile, an all-optical two-color pump-probe technique utilizing weak attosecond XUV pulses and strong few-cycle NIR pulses-often referred to as attosecond transient absorption spectroscopy (ATAS)has opened a direct route to the measurement and control of the XUV spectral response of bound electronic transitions [13-21]. With both the XUV-NIR time delay $\tau$ and the NIR intensity $I_{N I R}$ as continuously tunable control parameters, ATAS has a multi-dimensional experimental anatomy [22]. Two-dimensional (2D) time-domain spectra $S(\tau, \omega)$ measured as a function of both the XUV photo-excitation frequency $\omega$ 
and time delay $\tau$ exhibit a characteristic fringe pattern across coherence resonances regardless of the specific target system [23]. The Fourier transform along the signal's pulse-delay time trace is carried out $(\tau \rightarrow v)$ to decompose the interference pattern into a twodimensional absorption spectrum (2DAS), $\tilde{S}(\nu, \omega)$, which exhibits diagonal regions with point-like and/or line-like peaks [13,18,22,24,25] revealing information about the dynamical pathways along which the system is driven. We recently introduced an analytical framework to understand and characterize the signatures of time-delay dependent polarization dynamics in 2D-XUV absorption spectra [23].

Thus far, in ATAS studies only the dipole-allowed transitions were excited by a single-photon XUV pump step. Accordingly, the interference structures observed in the 2DAS only consisted of maxima at zero or even multiples of the NIR-photon energy. Interferences at odd multiples of the NIR-photon energy would indicate couplings to additional (one-photon forbidden) channels, which could be accessed by a combination of XUV and NIR excitation fields, as previously applied to photo-electron spectroscopy [26,27].

Here we extend ATAS (typically a VUV/XUV and a single timedelayed laser pulse) into a time-resolved FWM technique, where excitation and probing by an NIR laser pulse occurs at two different times. As we will show, this allows the spectroscopy on both dipole-allowed and -forbidden XUV-excited inner-valence transitions. The first two pulses (XUV and weak NIR) coincide in time (with locked zero-delay timing and a fixed phase relation between fundamental field and higher harmonics) and are temporally separated from the strong (nonperturbative) third (NIR) pulse by the time delay $\tau$. The interaction of a sample with the first two pulses (XUV and NIR) creates a polarization, i.e., the coherent superposition of ground and both odd- and evenparity excited states. This two-color pump step extends the coherent excitation onto states the transition into which would be forbidden by a single XUV photon from the ground state. After the excited system has evolved freely during the time delay $\tau$, the interaction with the strong NIR pulse generates a nonlinear (third-order) response signal by again coupling between XUV-dipole-allowed and -forbidden excited states. We have chosen the $2 s$-inner-valence excited states $2 s^{-1} 3 s\left({ }^{1} \mathrm{~S}\right), 2 s^{-1} 3 d\left({ }^{1} \mathrm{D}^{\mathrm{e}}\right)$ and $2 s^{-1} 3 p\left({ }^{1} \mathrm{Po}^{\mathrm{o}}\right)$ of neon (henceforth denoted as $3 s, 3 d$, and $3 p$ ) as a target to perform first such proof-ofprinciple FWM experiments. Neon provides an appropriate electronic energy-level structure for investigating resonant transitions between XUV-dipole-allowed and -forbidden states which cannot be accessed by conventional ATAS methods.

The experimental setup (see Fig. 1a), as previously described in Ref. [17], involves a commercial Ti:Sapphire multipass amplifier with hollow-core fiber and chirped-mirror compression stages for the generation of NIR (central photon energy $\sim 1.6 \mathrm{eV}$ ) sub-7 fs pulses delivered at a $4 \mathrm{kHz}$ repetition rate and $\sim 0.4 \mathrm{~mJ}$ pulse energy. These pulses act as driver pulses for HHG in an argon gas-filled cell ( $\sim 75$ mbar backing pressure) yielding short trains of attosecond pulses that are inherently phase-locked to the fundamental field. HHG spectra obtained extend continuously over the autoionizing resonance region of neon between $43 \mathrm{eV}$ and $50 \mathrm{eV}$ covering the full $2 s^{-1} n p$ Rydberg series (cf. the HHG absorption spectrum in Fig. 1b). A piezo-driven two-component split mirror (inner, dynamic part: gold coating; outer part: silver coating) is employed to introduce a time delay between the co-propagating attosecond XUV and femtosecond NIR pulses. The NIR pulse intensity is controlled by a piezo-controlled iris aperture. Spatial beam separation between XUV and NIR is achieved by a two-part spectral band-pass filter in annular geometry where the outer, annular, part consists of a $2 \mu \mathrm{m}$ thin nitrocellulose membrane (1.2 mm central hole) and the inner part is a single layer of $0.2 \mu \mathrm{m}$ thin aluminum foil (1.2 mm diameter). Importantly, for the results shown in this work, the central part transmits a residual NIR-

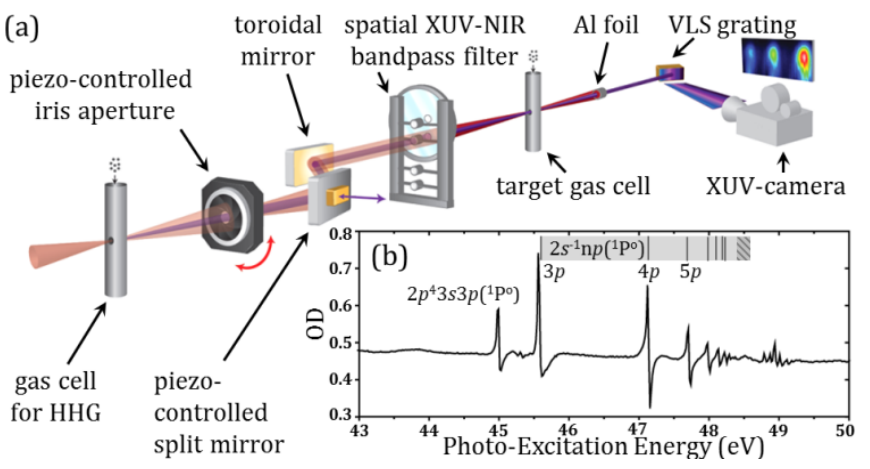

Fig. 1. (a) Schematic of the experimental setup and the key elements, as discussed in the main text. Essential for creating the described pulse configuration for FWM (temporally locked XUV-NIR excitation fields and NIR probe field) is the piezo-controlled split mirror in combination with an annular XUV-NIR filter geometry transmitting a residual NIRphoton flux through the inner Al filter. (b) Linear spectroscopy (XUV only): The natural neon line spectrum in terms of optical densities (OD). For more details on the experimental data acquisition, see Ref. [16]. Experimental resonances are assigned based on Ref. [38].

photon intensity on the order of $1-10 \%$, which can be considered as a perturbative NIR replica pulse which remains locked in temporal overlap with the XUV. The NIR transmission is due to small holes or other imperfections in the extremely fragile foil (e.g. created during the manufacturing process and/or mechanical strain while assembling the custom-made filter arrangement). After passing the band-pass filter, the NIR-pulse delay (with respect to the NIR-XUV excitation step) and intensity-controlled laser beams are focused into the neon gas-filled target cell $(\sim 35$ mbar backing pressure). The transmitted XUVlight contains the sample's dipole response which is detected by a high-resolution XUV spectrometer $(\sim 20 \mathrm{meV}$ Gaussian standard deviation near $45 \mathrm{eV}$, see Ref. [16] for details).

For comparison with our experimental results, calculations based on the R-matrix with time-dependence (RMT) approach [28] were performed for the neon atom. RMT is an $a b$ initio, non-perturbative computational technique for the description of general multi-electron atomic/ionic systems interacting with a strong laser field. By employing the standard R-matrix division of space [29] a tractable solution of the time-dependent Schrödinger equation can be obtained affording a full account of multi-electron effects, and a comprehensive treatment of detailed atomic structure. In order to describe both the core- and doubly-excited states of neon under investigation, the calculations comprise all single-, double- and triple-excitations of the $2 s$ and $2 p$ electrons into $3 p, 3 s, 3 d$, (spectroscopic) $\overline{4 s}$ and $\overline{4 p}$ (pseudo)-orbitals, which are determined as described in Ref. [30]. This leads to an expansion over 152 multi-electron configurations. Additionally, to account for the various ionization pathways, six residual ion states of $\mathrm{Ne}^{+}$are included in the calculation. This yields energies within $0.08 \mathrm{eV}$ of the literature values, for the states under investigation [31-33]. Using RMT we compute $\boldsymbol{d}(t)$ : the timedependent expectation value of the dipole operator. The absorption spectrum is then given by [34]

$$
\sigma(\omega)=4 \pi \frac{\omega}{c} \operatorname{Im}\left[\frac{d(\omega)}{E(\omega)}\right]
$$

where $\omega$ is the photon energy, $c$ the speed of light, and $\boldsymbol{d}(\omega)$ and $\boldsymbol{E}(\omega)$ are the Fourier transform of $\boldsymbol{d}(t)$ and the electric field, respectively.

In Figs. 2(a) and (2b), we present time-delay-resolved absorption measurements for a scanned time delay $\tau$ between XUV-NIR pump and NIR-probe fields, obtained from experiment and RMT calculations. Time-resolved absorption changes can be observed across all the reso- 


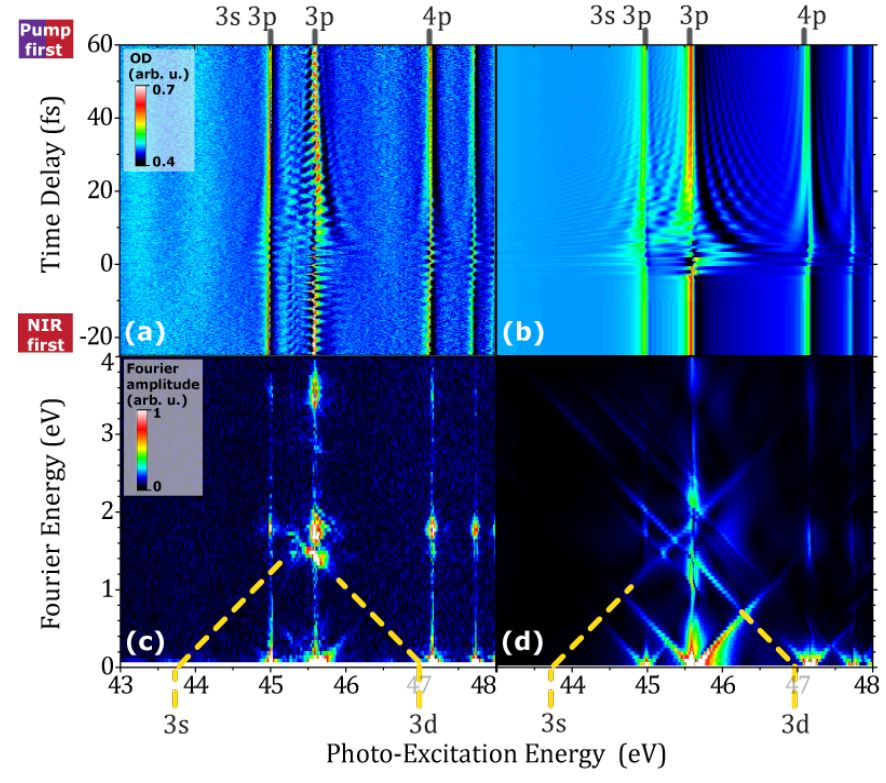

Fig. 2. Time-resolved 2D absorption spectra $S(\tau, \omega)$ at $\sim 10^{12} \mathrm{~W} / \mathrm{cm}^{2}$ NIR-probe intensity obtained from (a) experiment and (b) full $a b$ initio calculations assuming Gaussian pulses and for the NIR-pump $10 \%$ of the NIR-probe intensity (also see Dataset 1 in Ref. [43]). Strong periodic fullcycle modulations are imprinted on the $3 p$ resonance which give rise to diagonal, line-like peaks at $\sim 1.6 \mathrm{eV}$ Fourier energy in the 2DAS, $\tilde{S}(\nu, \omega)$ (lower panels c and d, respectively), indicating coherent coupling pathways. Vertically aligned peaks are due to time-delay dependent NIR intensity variations in the temporal overlap region. The energetic position of the dipole-forbidden and thus spectroscopically hidden $3 s$ and $3 d$ coupling partners $\left(\hbar \omega_{3 s}=43.7 \mathrm{eV}\right.$ and $\left.\hbar \omega_{3 d}=47.0 \mathrm{eV}\right)$ are indicated by the dashed lines as a guide to the eye. Besides the excellent agreement between experiment and RMT calculations, remaining discrepancies are likely due to uncertainties in the exact experimental pulse shapes. The $3 s$ and $3 d$ resonances are assigned based on Ref. [32].

nance lines shown in Figs. 2(a) and 2(b). Our focus here is on the absorption changes occurring near the $3 p$ state at $\hbar \omega_{3 p}=$ $45.55 \mathrm{eV}$ photo-excitation energy in order to understand the twophoton XUV-NIR-induced wave-packet dynamics created by the superposition of two coherently excited states with different parities. The time-domain absorption spectra $S(\tau, \omega)$ (Figs. 2a and 2b) are Fourier analyzed $(\tau \rightarrow v)$ to decompose the temporal beat patterns for each resonance into distinct peaks on a $2 \mathrm{DAS}, \tilde{S}(v, \omega)$, spectral representation (Figs. $2 \mathrm{c}$ and $2 \mathrm{~d}$ ). Peak position, shape, and orientation allow the characterization of coherent coupling and transition pathways analogous to the case previously described for XUV-only excitation and a single time-delayed NIR probe pulse [23]. For the case presented here, the additional weak NIR pulse locked to the XUV excitation pulse near time zero plays a crucial role, as it coherently excites states that would otherwise be dipole-forbidden for an XUV-only excitation. The basic structures observed in the time-domain spectra $S(\tau, \omega)$ (Figs. 2a and $2 b$ ) have been seen in several related ATAS studies with XUV-onlyexcitation fields [13-19,22,23,34-37] and can in general be decoupled into two interfering sub-structures [23]: $(i)$, a slow, $\tau$-dependent modulation following a hyperbolic geometry near resonance lines corresponds to a fork-like feature with elliptically-shaped peaks with slope 1 originating at zero Fourier energy in the 2DAS $\tilde{S}(v, \omega)$. (ii), a fast $\tau$-dependent rippling-type pattern, which gives rise to line-like Fourier peaks of slope 1 at non-zero Fourier energies. According to dipole-selection rules such Fourier peaks at even-numbered multiples of the NIR-photon energy indicate transitions between identical-parity
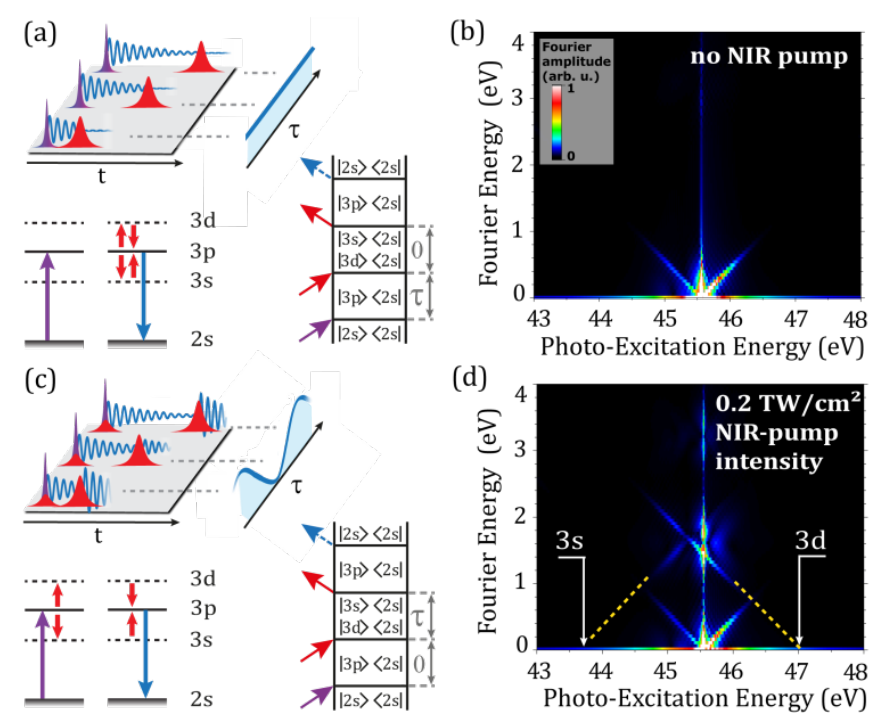

Fig. 3. Schematic illustrations and Feynman diagrams of the $\tau$-dependent third-order coupling mechanisms (panels a and c) and corresponding few-level model simulations (panels b and d). (a) The XUV pulse excites an $2 s$ electron (violet arrow) creating a coherent superposition of the ground state and the $3 p$ state while XUV transitions into final states $3 s$ and $3 d$ are dipole-forbidden. The superposition leads to coherent dipole emission (blue wavy lines). After the time delay $\tau$ the NIR pulse induces transient coupling (red arrows) which transfers population out of the $3 p$ into $3 s$ and $3 d$ states. Amplitude and phase modifications lead to the characteristic spectral features near zero Fourier energy in the 2DAS (panel b). (c) Two-photon (XUV-NIR) excitation into a coherent superposition of $3 p, 3 s$, and $3 d$ states. After the time delay $\tau$, during which the superposition has freely evolved in time, the interaction with the third (NIR) pulse mediates the exchange of population between these states which leads to constructive or destructive dipole emission of the $3 p$ state periodically with the optical period of the NIR pulse as a function of time delay. In the 2DAS (panel d) this creates the line-like features near a Fourier energy corresponding to the NIR photon energy.

states mediated by an even number of NIR photons. Such features (half- and quarter-cycle modulations) have been observed and interpreted recently [13,18,22]. In Figs. 2(c) and 2(d) we observe these Fourier features, however, at one NIR-photon energy, $\sim 1.6 \mathrm{eV}$ (fullcycle modulations), evidencing a coherent excitation and timedependent coupling of opposite-parity states. We identify the evenparity $3 s$ and $3 d$ states as the coupling partners of the $3 p$ state by analyzing the orientation of the two dominant line-like peaks appearing at $\hbar \omega_{3 p}=45.55 \mathrm{eV}$ photo-excitation energy and $\sim 1.6 \mathrm{eV}$ Fourier energy, which extrapolate towards the coupled resonance energies on the photo-excitation energy axis at $\hbar \omega_{3 s}=43.7 \mathrm{eV}$ and $\hbar \omega_{3 d}=47.0 \mathrm{eV}$ [32]. It is important to note that the two-color XUVNIR pump step for the coherent excitation of both XUV-dipole-allowed and dipole-forbidden (allowed by the absorption of an additional NIR photon near time zero) transitions to final states $3 p$ and $3 s, 3 d$ is the key mechanism to observing Fourier features at odd-numbered multiples of the NIR-photon energy. To further confirm this understanding we present in Fig. 3 few-level model (FLM) simulations based on the approach previously described in Ref. [16] assuming $2 \times 10^{11}$ $\mathrm{W} / \mathrm{cm}^{2}$ NIR-pump and $2 \times 10^{12} \mathrm{~W} / \mathrm{cm}^{2}$ NIR-probe intensities. The numerical parameters (resonance energies, line widths, asymmetry $q$-parameters) used to model the three states $(3 p, 3 s, 3 d)$ above the ground state are taken from Refs. [32,38]. The $N=1$ ionization conti- 
nuum is not included, but we account for line-shape asymmetry effects due to continuum coupling [39] by the phase shift $\varphi(q)=2 \arg (q-$ $i)$ of the time-dependent dipole response function [17]. The dipolematrix elements were estimated based on hydrogen-like orbitals including a semi-empirical shielding of the nuclear charge by the partially filled inner shell [40]. We note the good agreement of the FLM simulation (Fig. 3d) with both the experimental and the RMT spectra shown in Fig. 2 which further substantiates our understanding of the underlying mechanism as a third-order (four-wave mixing) interaction process with three excited resonances $(3 p, 3 s, 3 d)$. In Fig. 3 we additionally present a schematic view of the discussed mechanism and the corresponding Feynman diagrams. In principle, this method allows the extraction of coupling strengths (dipole-matrix elements) from the experimental data, as soon as the pulse shape and intensity of the NIR pulse can be accurately determined [41].

In conclusion, we implemented the well-established two-pulse technique of ATAS in a novel four-wave-mixing scheme, where the excitation and probing by an NIR pulse occurs at different times in order to access excitation and coupling channels that otherwise would be spectroscopically hidden. This enabled a direct access to coupling dynamics between inner-valence excited states of opposite parity in neon. The FWM mechanism that underlies these dynamics was interpreted and fully understood on the basis of few-level model simulations. To account for the electronic correlation of the neon atom in full dimensionality we compared our experimental results with RMT calculations and found excellent agreement. This renders the presented methodology a promising experimental tool to investigate polarizabilities in multielectron systems [42] even in excited and metastable states of different parity on ultrafast timescales. Additionally, the method can be extended to more complex systems, including the site-specific probing of intramolecular electron dynamics and excitation transfer.

Funding. Deutsche Forschungsgemeinschaft (DFG) (PF 790/1-1); European Research Council (ERC) (X-MuSiC-616783); U.K. EPSRC (EP/G055416/1) CORINF under the Marie Curie Action of the European Commission. This work used the ARCHER UK National Supercomputing Service.

\section{References}

1. C. K. N. Patel and R. E. Slusher, Phys. Rev. Lett. 20, 1087 (1968).

2. M. Schmitt, G. Knopp, A. Materny, and W. Kiefer, Chem. Phys. Lett. 270, 9 (1997).

3. T. S. Rose and M. D. Fayer, Chem. Phys. Lett. 117, 12 (1985)

4. S. Mukamel, Principles of Nonlinear Optical Spectroscopy (Oxford University Press, 1999).

5. D. M. Jonas, Annu. Rev. Phys. Chem. 54, 425 (2003).

6. O. Golonzka, M. Khalil, N. Demirdöven, and A. Tokmakoff, Phys. Rev. Lett. 86, 2154 (2001).

7. T. Brixner, J. Stenger, H. M. Vaswani, and M. Cho, Nature 434, 625 (2005).

8. A. Halpin, P. J. M. Johnson, R. Tempelaar, R. S. Murphy, J. Knoester, T. L. C. Jansen, and R. J. D. Miller, Nat. Chem. 6, 196 (2014).

9. I. V. Schweigert and S. Mukamel, Phys. Rev. A 76, 012504 (2007).

10. I. V. Schweigert and S. Mukamel, Phys. Rev. Lett. 99, 163001 (2007).

11. E. Sistrunk, J. Grilj, J. Jeong, M. G. Samant, A. X. Gray, H. A. Dürr, S. S. P. Parkin, and M. Gühr, Opt. Express 23, 4340 (2015)

12. F. Bencivenga, R. Cucini, F. Capotondi, A. Battistoni, R. Mincigrucci, E. Giangrisostomi, A. Gessini, M. Manfredda, I. P. Nikolov, E. Pedersoli, E. Principi, C. Svetina, P. Parisse, F. Casolari, M. B. Danailov, M. Kiskinova, and C. Masciovecchio, Nature 520, 205 (2015).

13. M. Chini, X. Wang, Y. Cheng, Y. Wu, D. Zhao, D. a Telnov, S.-I. Chu, and Z. Chang, Sci. Rep. 3, 1105 (2013).

14. B. Bernhardt, A. R. Beck, X. Li, E. R. Warrick, M. J. Bell, D. J. Haxton, C.
W. McCurdy, D. M. Neumark, and S. R. Leone, Phys. Rev. A 89, 023408 (2014).

15. L. Gallmann, J. Herrmann, R. Locher, M. Sabbar, A. Ludwig, M. Lucchini, and U. Keller, Mol. Phys. 111, 2243 (2013).

16. C. Ott, A. Kaldun, L. Argenti, P. Raith, K. Meyer, M. Laux, Y. Zhang, A. Blättermann, S. Hagstotz, T. Ding, R. Heck, J. Madroñero, F. Martín, and T. Pfeifer, Nature 516, 374 (2014).

17. C. Ott, A. Kaldun, P. Raith, K. Meyer, M. Laux, J. Evers, C. H. Keitel, C. H. Greene, and T. Pfeifer, Science 340, 716 (2013).

18. C. Ott, A. Kaldun, P. Raith, K. Meyer, M. Laux, Y. Zhang, S. Hagstotz, T. Ding, R. Heck, and T. Pfeifer, arXiv:1205.0519 (2012).

19. H. Wang, M. Chini, S. Chen, C. H. Zhang, F. He, Y. Cheng, Y. Wu, U. Thumm, and Z. Chang, Phys. Rev. Lett. 105, 143002 (2010).

20. M. Holler, F. Schapper, L. Gallmann, and U. Keller, Phys. Rev. Lett. 106, 123601 (2011).

21. E. Goulielmakis, Z.-H. Loh, A. Wirth, R. Santra, N. Rohringer, V. S. Yakovlev, S. Zherebtsov, T. Pfeifer, A. M. Azzeer, M. F. Kling, S. R. Leone, and F. Krausz, Nature 466, 739 (2010).

22. L. Argenti, Á. Jiménez-Galán, C. Marante, C. Ott, T. Pfeifer, and F. Martín, Phys. Rev. A 91, 061403 (2015).

23. A. Blättermann, C. Ott, A. Kaldun, T. Ding, and T. Pfeifer, J. Phys. B: At. Mol. Opt. Phys. 47, 124008 (2014).

24. J. Mauritsson, T. Remetter, M. Swoboda, K. Klünder, A. L'Huillier, K. J. Schafer, O. Ghafur, F. Kelkensberg, W. Siu, P. Johnsson, M. J. J. Vrakking, I. Znakovskaya, T. Uphues, S. Zherebtsov, M. F. Kling, F. Lépine, E. Benedetti, F. Ferrari, G. Sansone, and M. Nisoli, Phys. Rev. Lett. 105, 053001 (2010).

25. M. Lucchini, A. Ludwig, T. Zimmermann, L. Kasmi, J. Herrmann, A. Scrinzi, A. S. Landsman, L. Gallmann, and U. Keller, Phys. Rev. A 91, 063406 (2015).

26. N. Shivaram, H. Timmers, X. M. Tong, and A. Sandhu, Phys. Rev. Lett. 108, 193002 (2012).

27. P. Ranitovic, X. M. Tong, B. Gramkow, S. De, B. DePaola, K. P. Singh, W. Cao, M. Magrakvelidze, D. Ray, I. Bocharova, H. Mashiko, A. Sandhu, E. Gagnon, M. M. Murnane, H. Kapteyn, I. Litvinyuk, and C. L. Cocke, New J. Phys. 12, 013008 (2010).

28. L. R. Moore, M. A. Lysaght, L. A. A. Nikolopoulos, J. S. Parker, H. W. van der Hart, and K. T. Taylor, J. Mod. Opt. 58, 1132 (2011).

29. P. G. Burke and K. A. Berrington, Atomic and Molecular Processes : An R-Matrix Approach (IOP, Bristol, 1993).

30. K. Schulz, M. Domke, R. Püttner, A. Gutiérrez, G. Kaindl, G. Miecznik, and C. Greene, Phys. Rev. A 54, 3095 (1996).

31. A. Kramida, Y. Ralchenko, J. Reader, and NIST ASD Team (2014), "NIST Atomic Spectra Database (version 5.2)," Available: http://physics.nist.gov/asd [2015, February 2]. National Institute of Standards and Technology, Gaithersburg, MD.

32. G. Min, Z. Lin-Fan, L. Cun-Ding, and X. Ke-Zun, Chinese Phys. Lett. 25, 3646 (2008).

33. D. Spence, J. Phys. B: At. Mol. Opt. Phys. 14, 129 (1999).

34. M. B. Gaarde, C. Buth, J. L. Tate, and K. J. Schafer, Phys. Rev. A 83, 013419 (2011).

35. X. Wang, M. Chini, Y. Cheng, Y. Wu, X. M. Tong, and Z. Chang, Phys. Rev. A 87, 063413 (2013).

36. S. Chen, M. Wu, M. B. Gaarde, and K. J. Schafer, Phys. Rev. A 87, 033408 (2013).

37. W. C. Chu and C. D. Lin, Phys. Rev. A 85, 013409 (2012).

38. K. Codling, R. P. Madden, and D. L. Ederer, 155, 26 (1967).

39. U. Fano, Phys. Rev. 124, 1866 (1961).

40. J. C. Slater, Phys. Rev. 36, 57 (1930).

41. A. Blättermann, C. Ott, A. Kaldun, T. Ding, V. Stooß, M. Laux, M. Rebholz, and T. Pfeifer, Opt. Lett. 40, 3464 (2015).

42. J. Mitroy, M. S. Safronova, and C. W. Clark, J. Phys. B: At. Mol. Opt. Phys. 43, 3464 (2010).

43. A. C. Brown, "Attosecond Transient Absorption of Neon," (2015), http://dx.doi.org/10.17034/fa84d593-d1f2-4580-8419-fc7997871a80 


\section{References}

1. C. K. N. Patel and R. E. Slusher, "Photon echoes in gases," Phys. Rev. Lett. 20, 1087 (1968).

2. M. Schmitt, G. Knopp, A. Materny, and W. Kiefer, "Femtosecond timeresolved coherent anti-Stokes Raman scattering for the simultaneous study of ultrafast ground and excited state dynamics: iodine vapour," Chem. Phys. Lett. 270, 9 (1997).

3. T. S. Rose and M. D. Fayer, "Probing gas-phase dynamics with picosecond transient grating spectroscopy," Chem. Phys. Lett. 117, 12 (1985)

4. S. Mukamel, Principles of Nonlinear Optical Spectroscopy (Oxford University Press, 1999).

5. D. M. Jonas, "Two-dimensional femtosecond spectroscopy," Annu. Rev. Phys. Chem. 54, 425-63 (2003).

6. O. Golonzka, M. Khalil, N. Demirdöven, and A. Tokmakoff, "Vibrational anharmonicities revealed by coherent two-dimensional infrared spectroscopy," Phys. Rev. Lett. 86, 2154-2157 (2001).

7. T. Brixner, J. Stenger, H. M. Vaswani, and M. Cho, "Two-dimensional spectroscopy of electronic couplings in photosynthesis," Nature 434, 625-628 (2005).

8. A. Halpin, P. J. M. Johnson, R. Tempelaar, R. S. Murphy, J. Knoester, T. L. C. Jansen, and R. J. D. Miller, "Two-dimensional spectroscopy of a molecular dimer unveils the effects of vibronic coupling on exciton coherences," Nat. Chem. 6, 196-201 (2014).

9. I. V. Schweigert and S. Mukamel, "Probing valence electronic wavepacket dynamics by all x-ray stimulated Raman spectroscopy: A simulation study," Phys. Rev. A 76, 012504 (2007)

10. I. V. Schweigert and S. Mukamel, "Coherent Ultrafast Core-Hole Correlation Spectroscopy: X-Ray Analogues of Multidimensional NMR," Phys. Rev. Lett. 99, 163001 (2007).

11. E. Sistrunk, J. Grilj, J. Jeong, M. G. Samant, A. X. Gray, H. A. Dürr, S. S. P. Parkin, and M. Gühr, "Broadband extreme ultraviolet probing of transient gratings in vanadium dioxide," Opt. Express 23, 4340 (2015)

12. F. Bencivenga, R. Cucini, F. Capotondi, A. Battistoni, R. Mincigrucci, E. Giangrisostomi, A. Gessini, M. Manfredda, I. P. Nikolov, E. Pedersoli, E. Principi, C. Svetina, P. Parisse, F. Casolari, M. B. Danailov, M. Kiskinova, and C. Masciovecchio, "Four-wave mixing experiments with extreme ultraviolet transient gratings," Nature 520, 205-208 (2015).

13. M. Chini, X. Wang, Y. Cheng, Y. Wu, D. Zhao, D. a Telnov, S.-I. Chu, and Z. Chang, "Sub-cycle oscillations in virtual states brought to light," Sci. Rep. 3, 1105 (2013).

14. B. Bernhardt, A. R. Beck, X. Li, E. R. Warrick, M. J. Bell, D. J. Haxton, C. W. McCurdy, D. M. Neumark, and S. R. Leone, "High-spectral-resolution attosecond absorption spectroscopy of autoionization in xenon," Phys. Rev. A - At. Mol. Opt. Phys. 89, 023408 (2014).

15. L. Gallmann, J. Herrmann, R. Locher, M. Sabbar, A. Ludwig, M. Lucchini, and U. Keller, "Resolving intra-atomic electron dynamics with attosecond transient absorption spectroscopy," Mol. Phys. 111, 22432250 (2013).

16. C. Ott, A. Kaldun, L. Argenti, P. Raith, K. Meyer, M. Laux, Y. Zhang, A. Blättermann, S. Hagstotz, T. Ding, R. Heck, J. Madroñero, F. Martín, and T. Pfeifer, "Reconstruction and control of a time-dependent twoelectron wave packet," Nature 516, 374-378 (2014).

17. C. Ott, A. Kaldun, P. Raith, K. Meyer, M. Laux, J. Evers, C. H. Keitel, C. H. Greene, and T. Pfeifer, "Lorentz Meets Fano in Spectral Line Laser Control," Science 340, 716-720 (2013).

18. C. Ott, A. Kaldun, P. Raith, K. Meyer, M. Laux, Y. Zhang, S. Hagstotz, T. Ding, R. Heck, and T. Pfeifer, "Quantum Interferometry and Correlated Two-Electron Wave-Packet Observation in Helium," arXiv:1205.0519 (2012).

19. H. Wang, M. Chini, S. Chen, C. H. Zhang, F. He, Y. Cheng, Y. Wu, U. Thumm, and Z. Chang, "Attosecond time-resolved autoionization of argon," Phys. Rev. Lett. 105, 143002 (2010).
20. M. Holler, F. Schapper, L. Gallmann, and U. Keller, "Attosecond Electron Wave-Packet Interference Observed by Transient Absorption," Phys. Rev. Lett. 106, 123601 (2011).

21. E. Goulielmakis, Z.-H. Loh, A. Wirth, R. Santra, N. Rohringer, V. S. Yakovlev, S. Zherebtsov, T. Pfeifer, A. M. Azzeer, M. F. Kling, S. R. Leone, and F. Krausz, "Real-time observation of valence electron motion," Nature 466, 739-743 (2010).

22. L. Argenti, Á. Jiménez-Galán, C. Marante, C. Ott, T. Pfeifer, and F. Martín, "Dressing effects in the attosecond transient absorption spectra of doubly excited states in helium," Phys. Rev. A 91, 061403 (2015).

23. A. Blättermann, C. Ott, A. Kaldun, T. Ding, and T. Pfeifer, "Twodimensional spectral interpretation of time-dependent absorption near laser-coupled resonances," J. Phys. B: At. Mol. Opt. Phys. 47, 124008 (2014).

24. J. Mauritsson, T. Remetter, M. Swoboda, K. Klünder, A. L'Huillier, K. J. Schafer, O. Ghafur, F. Kelkensberg, W. Siu, P. Johnsson, M. J. J. Vrakking, I. Znakovskaya, T. Uphues, S. Zherebtsov, M. F. Kling, F. Lépine, E. Benedetti, F. Ferrari, G. Sansone, and M. Nisoli, "Attosecond Electron Spectroscopy Using a Novel Interferometric Pump-Probe Technique," Phys. Rev. Lett. 105, 053001 (2010).

25. M. Lucchini, A. Ludwig, T. Zimmermann, L. Kasmi, J. Herrmann, A. Scrinzi, A. S. Landsman, L. Gallmann, and U. Keller, "Anisotropic emission in quantum-beat spectroscopy of helium excited states, " Phys. Rev. A 91, 063406 (2015).

26. N. Shivaram, H. Timmers, X. M. Tong, and A. Sandhu, "AttosecondResolved Evolution of a Laser-Dressed Helium Atom: Interfering Excitation Paths and Quantum Phases," Phys. Rev. Lett. 108, 193002 (2015).

27. P. Ranitovic, X. M. Tong, B. Gramkow, S. De, B. DePaola, K. P. Singh, W. Cao, M. Magrakvelidze, D. Ray, I. Bocharova, H. Mashiko, A. Sandhu, E. Gagnon, M. M. Murnane, H. Kapteyn, I. Litvinyuk, and C. L. Cocke, "IRassisted ionization of helium by attosecond extreme ultraviolet radiation," New J. Phys. 12, 013008 (2010).

28. L. R. Moore, M. A. Lysaght, L. A. A. Nikolopoulos, J. S. Parker, H. W. van der Hart, and K. T. Taylor, "The RMT method for many-electron atomic systems in intense short-pulse laser light," J. Mod. Opt. 58, 1132-1140 (2011).

29. P. G. Burke and K. A. Berrington, Atomic and Molecular Processes : An R-Matrix Approach (IOP, Bristol, 1993)

30. K. Schulz, M. Domke, R. Püttner, A. Gutiérrez, G. Kaindl, G. Miecznik, and C. Greene, "High-resolution experimental and theoretical study of singly and doubly excited resonances in ground-state photoionization of neon," Phys. Rev. A 54, 3095-3112 (1996).

31. A. Kramida, Y. Ralchenko, J. Reader, and NIST ASD Team (2014), "NIST Atomic Spectra Database (version 5.2)," Available: http://physics.nist.gov/asd [2015, February 2]. National Institute of Standards and Technology, Gaithersburg, MD.

32. G. Min, Z. Lin-Fan, L. Cun-Ding, and X. Ke-Zun, "Optically Forbidden Excitations of 2s Electron of Neon Studied by Fast Electron Impact," Chinese Phys. Lett. 25, 3646-3648 (2008).

33. D. Spence, "Autoionising states of $\mathrm{Ne}$ in the energy range 41 to $52 \mathrm{eV}$ derived from near-threshold scattered-electron spectra," J. Phys. B: At. Mol. Opt. Phys. 14, 129-147 (1999).

34. M. B. Gaarde, C. Buth, J. L. Tate, and K. J. Schafer, "Transient absorption and reshaping of ultrafast XUV light by laser-dressed helium," Phys. Rev. A - At. Mol. Opt. Phys. 83, 013419 (2011).

35. X. Wang, M. Chini, Y. Cheng, Y. Wu, X. M. Tong, and Z. Chang, "Subcycle laser control and quantum interferences in attosecond photoabsorption of neon," Phys. Rev. A - At. Mol. Opt. Phys. 87, 063413 (2013).

36. S. Chen, M. Wu, M. B. Gaarde, and K. J. Schafer, "Quantum interference in attosecond transient absorption of laser-dressed helium atoms," Phys. Rev. A - At. Mol. Opt. Phys. 87, 033408 (2013). 
37. W. C. Chu and C. D. Lin, "Photoabsorption of attosecond XUV light pulses by two strongly laser-coupled autoionizing states," Phys. Rev. AAt. Mol. Opt. Phys. 85, 013409 (2012).

38. K. Codling, R. P. Madden, and D. L. Ederer, "Resonances in the PhotoIonization Continuum of $\mathrm{Ne} I$ (20-150 eV)," 155, 26-38 (1967).

39. U. Fano, "Effect of configuration Interaction on intensities and phase shifts," Phys. Rev. 124, 1866-1878 (1961).

40. J. C. Slater, "Atomic shielding constants," Phys. Rev. 36, 57-64 (1930).

41. A. Blättermann, C. Ott, A. Kaldun, T. Ding, V. Stooß, M. Laux, M. Rebholz, and T. Pfeifer, "In situ characterization of few-cycle laser pulses in transient absorption spectroscopy," Opt. Lett. 40, 3464-3467 (2015).

42. J. Mitroy, M. S. Safronova, and C. W. Clark, "Theory and applications of atomic and ionic polarizabilities," J. Phys. B: At. Mol. Opt. Phys. 43, 3464-3467 (2010).

43. A. C. Brown, "Attosecond Transient Absorption of Neon," (2015), http://dx.doi.org/10.17034/fa84d593-d1f2-4580-8419-fc7997871a80 